\title{
ESTADO DA ARTE EM TECNOLOGIAS DE CAPTAÇÃO DA ENERGIA SOLAR PARA FINS TÉRMICOS
}

\author{
G. O. PRADO ${ }^{1,2}$, T. F. ULHOA ${ }^{2}$, J.J.R. DAMASCENO ${ }^{1}$, L.G.M. VIEIRA ${ }^{1}$ \\ ${ }^{1}$ Universidade Federal de Uberlândia, Departamento de Engenharia Química \\ ${ }^{2}$ Universidade Federal do Triângulo Mineiro, Departamento de Engenharia de Produção \\ E-mail para contato: webgop@gmail.com
}

\begin{abstract}
RESUMO - O aumento na demanda social por energia apresenta-se à tona, principalmente, por fatores como a evolução tecnológica e o crescimento industrial e demográfico. As fontes tradicionais de energia, tais como o petróleo, o carvão e o gás produzem resíduos exauridos da combustão que são agentes poluidores e extremamente nocivos. Uma das opções energéticas sem resquícios residuais é a energia solar, que além de ser limpa, tem como vantagem o fato de ser renovável. Para obtê-la, faz-se necessário a conversão da radiação solar, o que pode ser feito de duas maneiras: a forma direta, quando há conversão de energia solar em energia elétrica ou a forma indireta, valendo-se das propriedades térmicas da radiação. Este trabalho tem como intuito o estudo de tecnologias de utilização térmica da energia solar utilizando-se, para isso, da análise de referenciais da área tanto para aquecedores solares no formato plano quanto para concentradores solares.
\end{abstract}

\section{INTRODUÇÃO}

A sociedade depende de energia. Seu uso é associado às transações financeiras, ao entretenimento, às comunicações, ao controle térmico, à agricultura, aos tratamentos e diagnósticos medicinais, à culinária, à segurança patrimonial, às indústrias manufatureiras, ao transporte, enfim, ao estilo contemporâneo de vida. Entretanto, as fontes energéticas tradicionais tais como o petróleo, o carvão e o gás carecem de substitutos principalmente por apresentarem agentes poluidores e nocivos em sua combustão, além de serem fontes esgotáveis, ou seja, não renováveis.

Em contrapartida, a energia limpa e renovável é candidata à solução energética de uma possível crise global e tem como representantes: a energia eólica, a energia geotérmica, a biomassa, a energia hidráulica, a maré motriz e a energia solar. Esta última pode ser convertida de duas formas: direta ou indiretamente. A conversão direta pode ser feita, por exemplo, através de painéis solares, que utilizam células fotovoltaicas, comumente compostas por Silício amorfo, para transformar a energia solar em elétrica. Contudo, o presente artigo, tem como intuito o estudo da captação indireta da energia solar, ou seja, utilizando as propriedades da energia térmica, o que pode ser feito utilizando equipamentos planos ou concentradores de raios solares.

\section{UTILIZAÇÃO TÉRMICA DA ENERGIA SOLAR}


O aproveitamento da energia térmica do sol pode ser feito utilizando equipamentos planos, tais como aquecedores solares de fins domésticos ou através de concentradores solares, que captam os raios solares e os concentra em uma região focal linear ou pontual. A seguir, serão apresentados modelos de captação do tipo plano e também de concentradores solares.

\subsection{Modelo De Coletor Solar Plano}

Este modelo consiste na adaptação de uma superfície plana, geralmente composta por uma camada translúcida, que absorve e armazena o calor como uma estufa. Dessa forma, o calor é transmitido para um mecanismo ou circuito absorvedor, pintado na cor preta, um isolante térmico entre a estrutura do coletor e a edificação de instalação é fundamental para evitar perdas convectivas e condutivas de calor. Os isolantes térmicos mais comuns nesse tipo de equipamento são a lã de vidro e o isopor, embora haja também pesquisas de utilização de materiais residuais como a casca de coco, proposto por Andoh et al. (2010).

A utilização desse modelo é vinculada principalmente, ao aquecimento de águas de banho, de piscinas e de lavagem de roupas. Neste campo, existem tanto aquecedores solares comerciais quanto aqueles que buscam uma solução de baixo custo, como os apresentados pelas pesquisas de Siqueira et al. (2011) e Verraci et al. (2008) que se utilizaram de materiais alternativos na construção, tais como o PVC.

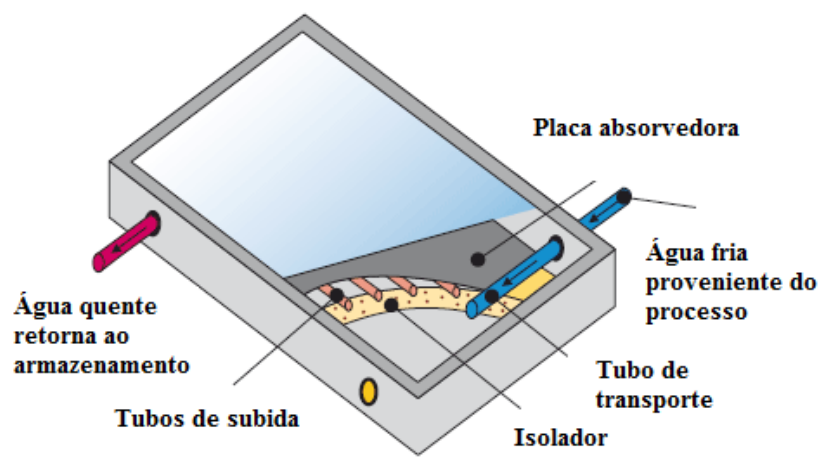

Figura 1 - Aquecedor Solar Plano, adaptado de Victoria Sustainability.

O sistema de aquecimento de água consiste em quatro passos distintos: a captação da energia solar, o aquecimento da água pelo coletor, o transporte da água entre o coletor e o reservatório e o armazenamento da água. O estudo de Penereiro et al. (2010) verificou a eficiência do coletores acoplando sensores termopares ao redor do coletor.

Por se tratar de um equipamento de instalação geralmente fixa a inclinação das placas coletoras também é uma variável e tem influencia direta na eficiência do modelo, sendo esta, relacionada à localização geográfica da instalação, conforme simulação para otimização feita por Stanciu e Stanciu (2014). Contudo, pode haver necessidade de uma resposta ainda mais eficiente no aquecimento solar, neste caso, uma estrutura de rastreamento do movimento solar seria o ideal, o que foi pesquisado por Neagoe et al. (2014).

\subsection{Modelo De Captação Solar Tipo Concentrador}


O concentrador solar é um equipamento construído com o intuito de direcionar os raios solares de forma pontual ou linear, utilizando-se dos princípios da reflexão ou da refração. Sua eficiência está principalmente vinculada à precisão óptica do equipamento coletor, às condições de isolamento térmico da parte absorvedora e às condições temporais do local de instalação, como vento, nuvens, chuva e níveis de incidência de radiação solar. Esta última, pode ser estimada através de ferramentas matemáticas e estatísticas tal como o modelo de Angström-Prescott (Almorox et al., 2008), ou medida, conforme cita Palz (2005), por piranômetros, heliógrafos e piro-neliômetros. Os tipos mais comuns de concentradores são: o coletor do tipo calha parabólica, do tipo bacia parabólica, a torre solar e as lentes Fresnel e são apresentados a seguir.

\subsubsection{Concentrador Solar Do Tipo Calha Parabólica}

O concentrador do tipo calha, cilíndrico ou calha-parabólica possui foco linear e um fator de concentração máximo de 212, conforme cita Duffie e Beckman (2013). A faixa de temperatura atingida pelo fluido aquecido varia usualmente entre $100{ }^{\circ} \mathrm{C}$ e $500{ }^{\circ} \mathrm{C}$, o que foi objeto de estudo de Mwesigye (2013), que relacionava também os valores de Entalpia. Este modelo de concentrador é utilizado como agente motriz de bombas para irrigação, em aquecimento de águas de caldeiras e em estações elétricas.

Seu funcionamento baseia-se na concentração de raios solares, pela superfície refletora, em um tubo receptor que é instalado no foco da parábola e que recebe o fluido a ser aquecido. Esse modelo geralmente vem acompanhado por um sistema de rastreamento solar e pode ser movimentado por apenas um eixo, no sentido Leste-Oeste, conforme ilustra a Figura 2.

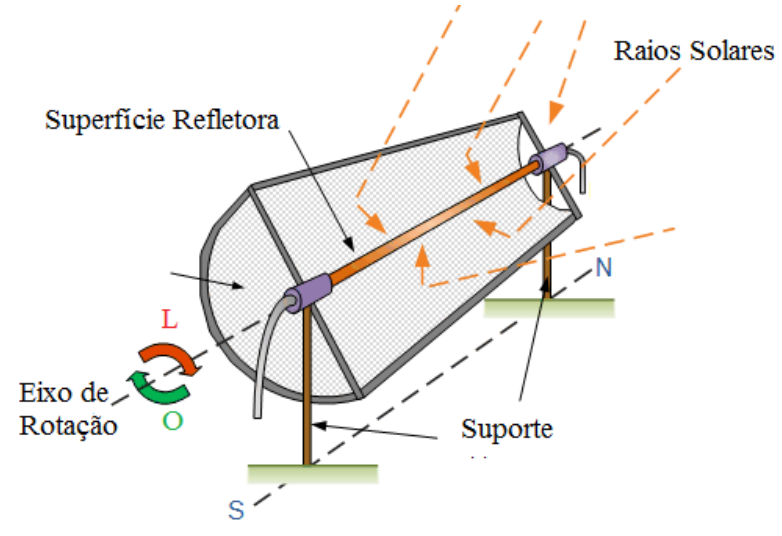

Figura 2- Concentrador tipo calha parabólica, adaptado de Alternative Energy Tutorials.

A redução no custo de fabricação desse tipo de projeto foi apresentado por Ho e Ito (2007), que tinham como meta um trabalho de inovação disruptiva, ou seja, visando um equipamento mais econômico embora com uma eficiência inferior. Sua estrutura principal foi construída a partir de madeira MDF, um tubo de cobre e uma superfície refletora feita de papel alumínio.

Assim como em outros modelos solares, uma das principais desvantagens desse tipo de tecnologia solar é o desamparo energético noturno. Para que isso seja suprido, utiliza-se de outros meios combustíveis para auxiliar na geração de energia. Segundo Lima et al. (2010), a 
energia solar é um dos modelos mais importantes quanto à obtenção de energia. Atualmente, porém, sua armazenagem deve ser de crucial importância ou gastos excessivos irão decorrer dos demais processos subsequentes.

\subsubsection{Concentrador Solar Tipo Bacia Parabólica}

Outro modelo de concentrador é o do tipo bacia parabólica, paraboloide ou parabólico de revolução, que possui foco pontual e tem um taxa de concentração máxima de 45000 , segundo cita Duffie e Beckman (2013). Sua utilização comum está vinculada à geração de energia elétrica, a fogões solares e a equipamentos que exigem altas temperaturas.

Seu funcionamento baseia-se na concentração de raios solares, pela superfície refletora, direcionando-os para um absorvedor pontual instalado no foco da parábola, ilustrado na Figura 3, que pode converter direta ou indiretamente a energia solar em elétrica. Para esse sistema, recomenda-se que haja um sistema de rastreamento solar acionado por dois eixos.

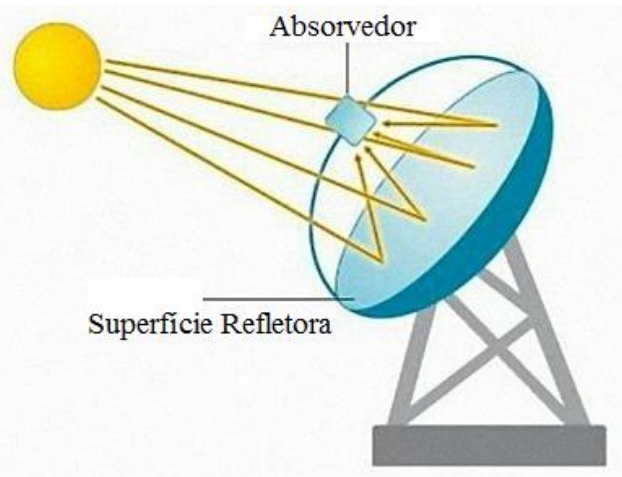

Figura 3-Concentrador solar do tipo bacia parabólica, adaptado de Energiverde.

No estudo de Lentz-Herrea et al. (2009), fora analisado a aplicação desse modelo para obtenção de vapor nas cidades mexicanas de Cierro Prieto e San Luis Rio Colorado, que possuem altos índices de irradiação e incidência solar. Já Villeda-Muñoz et al. (2011), elaborou um projeto de forno solar, com um aparato que mescla um concentrador solar a um heliostato com o intuito de direcionar os raios solares para um forno convencional. Nesse caso, o objetivo final era auxiliar na produção de tijolos de argila, o que resultaria em um impacto socioeconômico positivo nas comunidades carentes locais.

Pode-se considerar como desvantagem nos concentradores solares, a baixa produtividade associada ao tempo nublado, ou seja, quando a radiação solar torna-se difusa. Esse tipo de comportamento foi estudado e equacionado por Siala e Hooper (1990).

\subsubsection{Concentrador Solar Tipo Torre}

O modelo de concentrador do tipo torre é caracterizado por possuir superfícies reflexivas côncavas ou planas instaladas em um pilar comum e dotadas de um sistema de rastreamento solar de dois eixos. Elas circundam um absorvedor térmico fixo e localizado no topo de uma torre, de tal modo que os raios solares sejam direcionados e concentrados no absorvedor com faixa de operação de temperatura típica entre $100^{\circ} \mathrm{C}$ e $800^{\circ}$. O fluido térmico 
contido no absorvedor acionará um gerador mecânico de energia que funciona em um ciclo termodinâmico tal qual o de Rankine ou o de Brayton. A taxa de concentração usual alcançada é maior do que 1000 (IRENA, 2012).

Nesse tipo de captação de energia ocorre uma maior produção e consequentemente um maior armazenamento, o que garante um estoque para os períodos noturnos e dias com condições desfavoráveis. O armazenamento energético pode ser feito por um processo de aquecimento de sal, no qual, ele é aquecido atingindo altas temperaturas e estocado em tanques próximos a torre. Quando a utilização dessa energia armazenada é solicitada, o calor proveniente desse tanque é repassado ao gerador, e o sal resfriado é levado a outro tanque, para ser aquecido novamente.

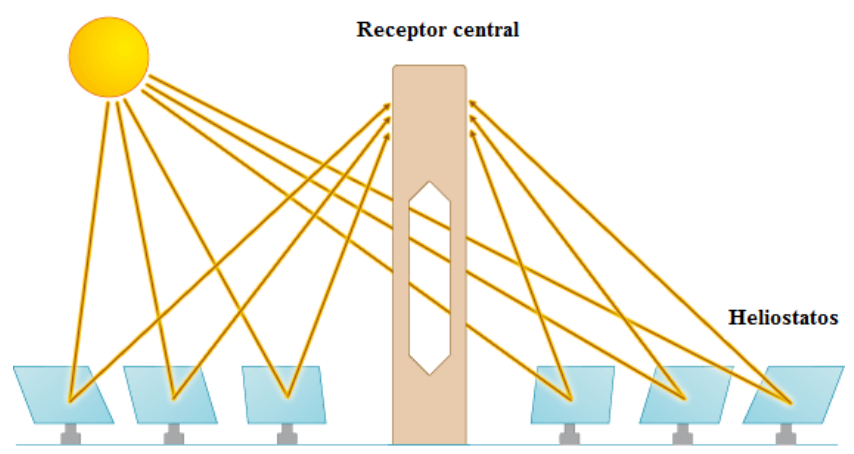

Figura 4- Torre solar em um campo de espelhos, adaptado de Abengoa Solar.

Riaz (1976) simulou os efeitos causados pelas sombras, incidência e o reflexo de radiação solar em uma planta ampla de concentradores solares do tipo torre. Já Ali (1990), propôs um modelo matemático para identificar as providências iniciais de uma planta energética tais como a altura da torre e a distância entre a torre e os espelhos heliostatos. Enquanto que Peterka et al. (1989) discute sobre as influências entre cargas de vento média e de pico em espelhos planos retangulares ou heliostatos circulares. E finalmente, Pitz-Pall, 2011 propõe uma configuração de layout para otimizar heliostatos, visando atingir altas temperaturas em processos termoquímicos.

\subsubsection{Concentrador Solar Por Refração}

Embora a maioria dos concentradores solares utilize sistemas baseados na reflexão, o modelo de lentes Fresnel, mais compactos que as lentes convencionais, utiliza-se dos princípios da refração. Nesse caso, o concentrador é composto por uma ou mais lentes que criam um ponto, uma linha ou uma área de concentração dos raios solares, conforme ilustrado na Figura 5, os quais são absorvidos na zona focal e repassados para a função desejada, que como nos outros casos, pode ser a geração de energia elétrica, o aquecimento de tubulações, a climatização de ambientes, etc.

Lara et al. (2012), utilizou este modelo de concentrador para o fornecimento de calor de um sistema de ar condicionado, afirmando que um modelo que utiliza as lentes Fresnel pode adquirir um alto nível de eficiência se otimizado corretamente. Já no estudo de Xie et al., 2013, a performance térmica de um sistema de foco linear foi testado considerando mudança de formato de receptores. 


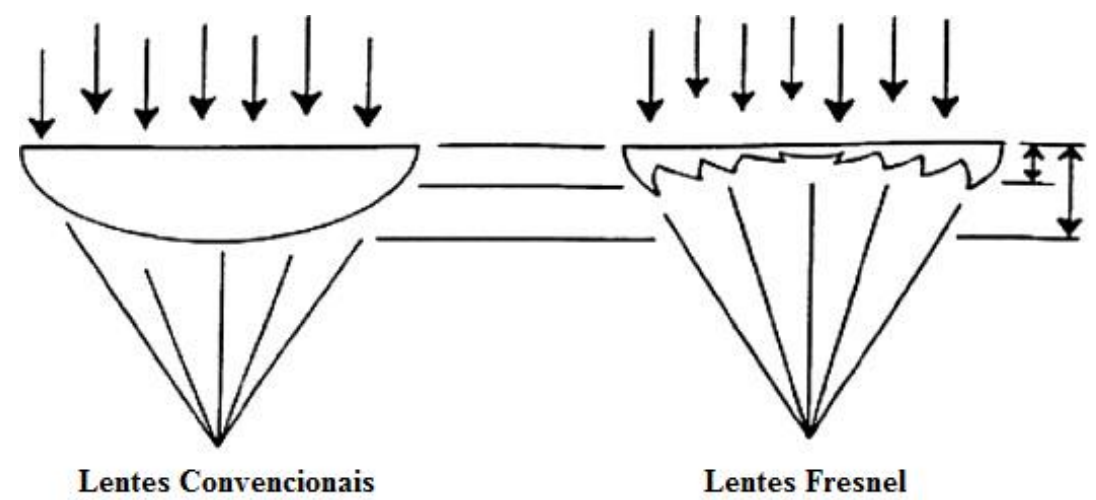

Figura 5- Comparativos de lentes, adaptado Xie et al. (2011).

\section{RESULTADOS E DISCUSSÕES}

A captação de energia solar na forma térmica possui diversas utilidades e várias formas de ser realizada. Sua eficiência (e) pode ser calculada conforme a Equação 1, em que $R$ representa a vazão mássica $\left[\mathrm{kg} \cdot \mathrm{s}^{-1}\right], C_{p}$ o calor específico $\left(\mathrm{J} \mathrm{kg}^{-1}\right)$ e $\left(\mathrm{T}_{\mathrm{s}}-\mathrm{T}_{\mathrm{e}}\right)$ a variação de temperatura entre a saída e a entrada, esses referentes ao fluido térmico, além de $G$ que representa a irradiação total incidente (W).

$$
e=\frac{R \cdot C_{p} \cdot\left(T_{S}-T_{e}\right)}{G}
$$

Entretanto, a eficiência do modelo depende de fatores como condições climáticas, área de captação e materiais utilizados na constituição do concentrador. No caso dos concentradores planos, de acordo com o estudo de Penereiro et al (2010), foram obtidos dados de eficiência equivalentes a aproximadamente $55 \%$, enquanto que em modelos mais complexos, atingiu-se $70 \%$ de eficiência relativa. Entretanto, o custo do projeto foi cerca de seis vezes menor, possibilitando a pesquisa de novas técnicas na área, envolvendo baixo custo e melhora na eficiência. Já no experimento de Ho e Ito (2007), foram obtidos valores de 14\% (dia nublado) e 19\% (dia ensolarado) para a eficiência do equipamento.

Nos concentradores tipo bacias parabólicas, o forno solar de Villeda-Muñoz et al. (2011) produziu 10 tijolos de argila por dia, porém se otimizado em maior escala, consegue chegar a uma produção de 110 tijolos/dia ou aproximadamente 3300 tijolos/mês. Para as as lentes Fresnel, o estudo de Lara et al. (2012) mostra que elas podem ser aplicadas em modelos para melhorar a eficiência de determinado processo obtendo dados de eficiência referentes a $82,4 \%$. E, por fim, no caso das torres solares, Pitz-Paal et al. (2011) otimizaram o potencial térmico de dois sistemas, um de óxido redução de zinco e outro de gaseificação de carvão, conseguindo uma taxa de conversão de energia solar para química $\left(e_{s-q}\right)$ de $30 \%$ e de $40 \%$, além das temperaturas de operação otimizadas de $1900 \mathrm{~K}$ e de $1300 \mathrm{~K}$, respectivamente. A Equação 2, ilustra o cálculo dessa taxa em que $H$ representa a entalpia específica de reação $\left(\mathrm{kJ} \mathrm{kg}^{-1}\right), I$ a irradiação normal direta $\left(\mathrm{W} . \mathrm{m}^{-2}\right), A$ a área refletida pelos heliostatos $\left(\mathrm{m}^{2}\right)$ e $v$ a taxa de reação $\left(\mathrm{kg} \cdot \mathrm{s}^{-1}\right)$.

$$
e_{S-q}=\frac{v H}{A I}
$$




\section{CONCLUSÃO}

A energia solar é uma das fontes energéticas mais cobiçadas na atualidade. Seu uso promove melhorias que alcançam diversos temas e camadas da sociedade, obtendo respaldo para o desenvolvimento de novas técnicas e a aplicação de outras tecnologias em conjunto. A sua captação e conversão, na forma térmica, foi objeto de estudo do presente artigo, nos casos sugeridos, foi apresentado sobre a eficiência em aquecedores solares planos e em concentradores do tipo calha parabólica de baixo-custo, a produção de tijolos utilizando um concentrador solar do tipo bacia parabólica, a otimização de layout de torres de heliostatos e, por fim, o trabalho de medidas de eficiência utilizando lentes Fresnel. Para continuar rumo ao sucesso, o empenho de esforços deve ser concentrado visando à sustentabilidade e a qualidade de vida para a sociedade, estudando meios energéticos com menores custos, mais limpos e, portanto, melhor para todos.

\section{AGRADECIMENTOS}

Os autores agradecem o apoio financeiro provido pela FAPEMIG (PCE-0020114: Participação Coletiva em Eventos Técnicos-Científicos).

\section{REFERÊNCIAS}

ALI, B.F. Theoretical study of main factors affecting the heliostat field design of tower power plant. Energy Conversion and Management, v. 30, p. 101-106, 1990.

ALMOROX, J.; BENITO, M.; HONTORIA, C. Estimation of global solar radiation in Venezuela. INCI, v. 33, 2008.

ANDOH, H.Y.; GBAHA, P.; KOUA, B.K.; KOFFI, P.M.E.; TOURÉ,S. Thermal performance study of a solar collector using a natural vegetable fiber, coconut coir, as heat insulation. Energy for Sustainable Development, v. 14, p.297-301, 2010.

IRENA. Renewable Energy Technologies: Cost Analysis Series. Volume 1: Power Sector. Alemanha: FSC, 2012.

DUFFIE, J.A; BECKMAN, W.A. Solar Engineering of Thermal Process. New York: Wiley, 2013.

HO, D. A.; ITO, E. A. Projeto e construção de aquecedor solar com concentrador. 2007. 51 f. Trabalho de Conclusão de Curso (Graduação em Engenharia Mecânica) - Faculdade de Engenharia Mecânica, Universidade de São Paulo, São Paulo, 2007.

LARA, F.; VELÁZQUEZ, N.; SAUCEDA, D.; ACUÑA, A. Metodología para el Dimensionamiento y Optimización de um Concentrador Lineal Fresnel. Información Tecnológica, v.24, p.115-128, 2013.

LENTZ-HERREA, A.; ALMANZA-SALGADO, R.; LÓPEZ-RIOS， S.; CADENASTOVAR, R. Available Energy and Cumulative Frequency Curves for Parabolic Trough Concentrators with Alignment North-South, in the Northwest of Mexico. RIIT, v.10, p.159166, 2009. 
NEAGOE, M..;VISA, I.; BURDUHOS, B.G.;MOLDOVAN, M.D. Thermal Load based Adaptive Tracking for Flat Plate Solar Collectors. Energy Procedia, v. 48, p. 1401-1411, 2014.

MWESIGYE, A.; BELLO-OCHENDE, T.; MEYER, J.P. Numerical investigation of entropy generation in a parabolic trough receiver at different concentration ratios. Energy, v. 53, p. 114-127, 2013.

PALZ, W. Energia Solar e Fontes Alternativas. Editora HEMUS, 2005.

PETERKA, J.A.; TAN, Z.; CERMAK, J.E; BIENKIEWICZ, B. Mean and peak wind loads on heliostats. Journal of Solar Energy Engineering, v.111, p. 158-164, 1989.

PENEREIRO, J.C.; MELO, L.P.; CORADI, T.B. Construção de um aquecedor solar de baixo custo sem cobertura: análise experimental da eficiência térmica para vários ensaios. Revista de Ciência \& Tecnologia, v.10, p.18-34, 2010.

PITZ-PALL, R.; BOTERO, N.B.; STEINFELD, A. Heliostat field layout optimization for high-temperature solar thermochemical processing. Solar Energy, v. 85, p. 334-343, 2011.

RIAZ, M.R.A. Theory of concentrators of solar energy on a central receiver for electric power generation. Journal of Engineering for Power, v. 83, p. 98-375, 1976.

SIALA, F.M; HOOPER, F.C.A model for the directional distribution of te diffuse sky radiance with an application to a cpc collector. Solar Energy, v. 44, p.291-296, 1990.

SIQUEIRA, D.A.; VIERA, L.G.M.; DAMASCENO, J.J.R. Analysis and performance of a low-cost solar heater. Renewable Energy, v.36, p. 2538-2546, 2011.

STANCIU, C.; STANCIU, D. Optimum tilt angle for flat plate collectors all over the World A declination dependence formula and comparisons of three solar radiation models. Energy Conversion and Management, v. 81, p. 133-143, 2014.

VILLEDA-MUÑOZ, G.; CASTAÑEDA-MIRANDA, A.; PLESS, R. C.; VEGA-DURÁN, J. T.; PINEDA-PIÑÓN, J. Clay-Brick Firing in a High-Temperature Solar Furnace. Ingeniería Investigación y Tecnología, v.12, p. 395-408, 2011.

VERRACI, M.H.; SILVA, F. C.; MOREIRA, A. P. S.; ANDRADE, G.M.; SALES, J. H. O.; SUZUKI, A. T. Painéis Solares do Tipo Plano e Parabólico. Anais do $14^{o}$ Encontro de Iniciação Científica e Pós-Graduação do ITA - XIV ENCITA, 2008.

LIMA, D. K. S.; MELO, F. D. C.; NETO, J. A. G.; MELO, C. M. N. Possíveis combustíveis auxiliares para uma usina solar térmica com concentradores parabólicos. VI CONGRESSO NACIONAL DE ENGENHARIA MECÂNICA, 2010.

XIE, W.T.; DAI,Y.J.; WANG, R.Z.; SUMATHY, K.Concentrated solar energy applications using Fresnel lenses: A review. Renewable and Sustainable Energy Review, v. 15, p. 25882606, 2011.

XIE, W.T.; DAI,Y.J.; WANG, R.Z. Thermal performance analysis of a linear collector using diferente cavity receivers. Solar Energy, v. 91, p. 242-255, 2013. 